\title{
Fatores críticos para a fixação do médico na Estratégia Saúde da Família
}

\section{| ${ }^{1}$ Márcia Silveira Ney, ${ }^{2}$ Paulo Henrique de Almeida Rodrigues |}

Resumo: O artigo analisa as condições que interferem na fixação do médico na Estratégia Saúde da Família. Trata-se de pesquisa de natureza qualitativa envolvendo análise documental das políticas de recursos humanos praticadas, aplicação e análise de questionários e grupo focal, visando identificar a percepção dos médicos sobre seu trabalho na estratégia. Revelou-se uma alta rotatividade dos médicos, determinada por insatisfação profissional, condições de trabalho inadequadas e carga de trabalho pesada. Dentre as características da política local de recursos humanos, destacaram-se distorções em relação à remuneração; e problemas no plano de cargos, carreira e salários em relação aos médicos de família, que limitam e penalizam a ascensão do profissional. Os principais motivos identificados na pesquisa que favorecem a permanência foram: identificação com a filosofia da estratégia, vocação profissional e possibilidade de servir à comunidade. São apresentadas reflexões sobre a gestão dos recursos humanos na estratégia e no SUS como um todo, apontando algumas das principais fragilidades da mesma, das quais se destacam: deficiências na política de formação profissional para a especialidade, insuficiente regulação da profissão médica no Brasil por parte do Estado e excessiva interferência política na prestação de serviços públicos de saúde, particularmente nessa estratégia.

> Palavras-chave: Estratégia Saúde da Família; medicina de família e comunidade; recursos humanos em saúde; políticas públicas de saúde.
1 Médica; mestre em Saúde da Família; doutoranda do Programa de Pós-graduação em Saúde Coletiva do IMS-UERJ. Endereço eletrônico: marcia. ney.unesa@gmail.com.

2 Professor adjunto do IMS-UER e do Mestrado em Saúde da Família da Universidade Estácio de Sá. Endereço eletrônico: phrodrigues@gmail.com
Recebido em: 13/12/2011. Aprovado em: 04/10/2012. 


\section{Introdução}

No panorama atual da política pública de saúde no Brasil, a Estratégia Saúde da Família, criada em 1994 como Programa Saúde da Família, é considerada pelo Ministério da Saúde (MS) uma estratégia de reorganização da atenção básica ou primária (BRASIL, 2006). Pode-se dizer que ela pretende promover a superação dos modelos assistenciais tradicionais, uma vez que sua proposta aponta para mudanças importantes nas práticas assistenciais, relações com as comunidades, organização das açōes e serviços, integração com os demais níveis de complexidade do sistema e forma de remuneração das ações de saúde.

Desde 1997, pelo menos, é apresentada pelo Ministério da Saúde como "uma estratégia para a reorientação do modelo assistencial" (BRASIL, 1997) como um todo e não apenas como de reorganização da atenção primária. Uma de suas principais inovações é o fato de estar voltada para comunidades e famílias em territórios definidos, a partir de ações desenvolvidas por equipes multiprofissionais, na qual se insere o médico de família (VIANA; DAL POZ, 1998). Tanto nos grandes centros urbanos, quanto nas áreas rurais, entretanto, a estratégia tem enfrentado dificuldades com relação à fixação do profissional médico nas suas equipes.

Estudo no município de São Paulo, realizado entre 2004 e 2005, de avaliação da existência de correlação entre satisfação no trabalho dos médicos e a rotatividade dos mesmos na ESF encontrou correlação negativa entre as duas variáveis (CAMPOS; MALIK, 2008, p. 361). Segundo o estudo, os fatores que apresentaram maior correlação com a rotatividade foram capacitação, distância até o local de trabalho e indisponibilidade de materiais para realizar o trabalho. Quanto à remuneração, a pesquisa indicou que o salário atrai os profissionais, mas não os fixa, e ainda seria uma forma de compensação de precariedade do vínculo (CAMPOS; MALIK, 2008, p. 362). Outros estudos confirmam a alta rotatividade do profissional médico como desafio para a expansão e qualificação das ações realizadas na estratégia'(GIRARDI;CARVALHO, 2003; BRASIL, 2005).

A gestão dos recursos humanos praticada no SUS e, portanto, na estratégia, vem sendo marcada, de um lado, pela política e regras de contenção dos gastos com pessoal estabelecidas pelo Governo Federal, e de outro, pela flexibilização dos vínculos de trabalho, que leva à precarização dos direitos trabalhistas. Em 
1995, um ano após a instituição da estratégia, foi promulgada a primeira Lei

Camata, que impôs limites para gastos com pessoal. Esta foi modificada em 1999 e consolidada pela Lei de Responsabilidade Fiscal de 2000, que vigora até hoje (BRASIL, 1995; 1999; 2000). Diversos autores mostram que, a partir da expansão da estratégia, os municípios se tornaram os principais responsáveis pela criação de postos de trabalho na saúde, e é nessa esfera de governo e na própria estratégia que tem se concentrado o problema dos vínculos precários de trabalho no SUS (GIRARDI; CARVALHO, 2002; JUNQUEIRA, 2010; MACIEL FILHO; PIERANTONI, 2004).

Outra questão relacionada à maior dificuldade para compor e manter o profissional médico nas Equipes de Saúde da Família (ESF) diz respeito à incipiente regulação estatal sobre a profissão médica no Brasil, ao contrário do que ocorre em outros países. Austrália, França, Reino Unido e Suécia, por exemplo, adotam políticas de regulação do estoque de médicos e suas especialidades através do controle da formação, por considerarem que o mercado de atenção à saúde não é um mercado livre (BLOOR; MAYNARD, 2003). Alguns países resolveram valorizar o papel da atenção primária nos seus sistemas de saúde e apresentavam, como resultado disso, elevada proporção de generalistas sobre o total de médicos, em 2005, tais como Austrália (52,1\%), França (49,3\%), Portugal (49\%) e Canadá (48\%), segundo a OECD (2011). A Espanha deu início a um amplo programa de residência para a formação de médicos de família sete anos antes da implantação do seu Sistema Nacional de Saúde; assim, quando começou a estruturar sua rede de atenção, onde as unidades de Atenção Primária desempenham papel chave, já contava com número expressivo de profissionais formados (FOSTER, 2004).

No Brasil, existe uma aparente rigidez no sistema de autorização e controle do exercício profissional, por meio de leis que estabelecem monopólios sobre campos de prática, mas não existem políticas voltadas para o controle do estoque das diferentes especialidades ou sua distribuição geográfica e formação, aspectos que têm a ver diretamente com o interesse público. Essa carência de regulação contribui para incentivar a formação de uma proporção elevada de especialistas, para a fragmentação da prática e para a insuficiência na oferta de generalistas. Tal situação influencia o deslocamento dos profissionais recém-formados para áreas em que o mercado oferece melhor remuneração e oportunidades 
de complementação da formação adquirida da graduação (SEIXAS, 2011). A definição de políticas relativas ao papel do médico de família como porta de entrada do SUS e à criação de condiçõoes para que esses especialistas representem uma proporção significativa do total de médicos, bem como um forte incentivo para sua formação, dependem evidentemente do Governo Federal. Os governos estaduais e municipais têm papel diminuto neste sentido.

No que diz respeito à gestão das ESF e, consequentemente, à manutenção dos médicos nas mesmas, há regras do Ministério da Saúde que têm de ser seguidas pelos municípios, para que não percam o acesso aos valores de incentivos para a implantação e o custeio das ESF (BRASIL, 2006). Constrangidos, de um lado, pelos limites de gastos com pessoal, e de outro, pela necessidade de ampliar a cobertura da Estratégia Saúde da Família e cumprir as regras do ministério, os municípios vêm utilizando uma série de mecanismos precários para a contratação de pessoal. Assim, em muitos municípios as regras adotadas para a gestão de recursos humanos na estratégia ferem tanto dispositivos da Constituição Federal, quanto os direitos trabalhistas. Tais expedientes precários afetam sobretudo os médicos, por serem os profissionais com salários mais altos e que os municípios vêm tendo maior dificuldade para atrair e manter na estratégia.

No Brasil, os médicos de família sentem-se sobrecarregados com a responsabilidade de prestar cuidados a um número muito grande de pessoas (até quatro mil pessoas), ao contrário do que ocorre em outros países que fixaram esse limite em duas mil pessoas, como é o caso de Portugal (PISCO, 2008) e da Espanha (GÉRVAS, 2005), ou muito menos, como é o caso de Cuba, onde o máximo são 400 pessoas (GOLDBERG, 2000). Outro agravante é que em muitos municípios do interior o médico de família é o único profissional médico que acolhe e trata a população, na maioria das vezes, extremamente carente, agravando sua sobrecarga de trabalho. Além disso, a precariedade de sistemas de referência, bem como dos serviços de apoio diagnóstico e tratamento, em muitos locais constitui um sério problema, que compromete a resolubilidade da atenção.

\section{Métodos}

A pesquisa procurou identificar os fatores críticos que interferem na fixação dos médicos na Saúde da Família, utilizando o município de Duque de 
Caxias como caso de estudo. Foi realizada como pré-requisito para a obtenção de titulação de Mestre em Saúde da Família na Universidade Estácio de Sá e contou com o financiamento de bolsa de estudos da Organização Panamericana de Saúde (OPAS).

Foram utilizados basicamente métodos qualitativos, que envolveram análise documental, realização de entrevistas e grupo focal. Para a verificação da rotatividade dos médicos na estratégia, foram analisados os dados das suas fichas funcionais, para se obter os dados de entrada e saída ano a ano, entre 2005 e 2008, sendo a análise feita através de distribuição de frequência simples. A análise documental visou analisar as políticas municipais de recursos humanos, utilizando documentos legais e documentos de reivindicação da categoria. Foi aplicado questionário semiestruturado, que constava de blocos temáticos buscando identificar: o perfil do profissional; a trajetória no mercado de trabalho; as considerações sobre as condições de trabalho na estratégia; e sua satisfação ou insatisfação com a função. Foram entrevistados 25 médicos de família, com mais de dois anos na estratégia. No período de realização do estudo, havia 51 médicos de família no município.

Foi realizado um grupo focal (GIL, 1994; CARLINI-COTRIM, 1996; HASSEN, 2002) com dez médicos de família que atuavam no município há pelo menos dois anos, selecionados aleatoriamente, entre os que preencheram o questionário. O roteiro do grupo focal teve como objetivo obter informações mais aprofundadas a respeito das percepções dos profissionais sobre o trabalho, enfocando as questôes extremamente necessárias para sua permanência na estratégia. Foram abordadas questões sobre condiçōes de trabalho, experiências vividas no cotidiano do trabalho, políticas de recursos humanos praticadas no município, mercado de trabalho atual e expectativas para o futuro. As falas e expressões foram transcritas e mantidas na sequência de argumentos e interpretação das mesmas. Para a análise das falas dos participantes, foi utilizada a metodologia com base no método de análise de conteúdo (BARDIN, 2007). A pesquisa foi submetida à apreciação do Comitê de Ética em Pesquisa (CEP) da Universidade Estácio de Sá, de acordo com a Resolução no. 196/96 do Conselho Nacional de Saúde (CNS) e em todas as fases realizadas foi utilizado o termo de consentimento livre e esclarecido. 


\section{Resultados}

\section{Políticas municipais de recursos humanos}

A implantação da estratégia no município ocorreu sem um planejamento adequado. As equipes foram formadas por profissionais que já atuavam em outras unidades de saúde, sendo convidados a migrar para a estratégia. Para tanto, foi aprovada uma lei municipal (DUQUE DE CAXIAS, 2001) que previa um regime de trabalho de $40 \mathrm{~h}$ com pagamento de gratificações específicas para a estratégia, não previstas no Estatuto dos Servidores Municipais. Como a lei municipal não assegurava a inclusão da gratificação no pagamento das férias, décimo-terceiro e licenças, isso motivou diversas reivindicaçōes desses profissionais.

Entre 2005 e 2008, com apoio do Programa de Expansão e Consolidação da Saúde da Família (PROESF) e os incentivos financeiros da Política Nacional de Atenção Básica e do Programa Brasil Sorridente (BRASIL, 2000; 2003) houve uma expansão do número de equipes da ordem de $82 \%$. Essa expansão exigiu a contratação temporária de um grande número de profissionais, que gerou dois regimes de trabalho diferentes na estratégia - estatutários com extensão de carga horária e contratados de forma temporária -, tal como acontece em muitos outros municípios do país (BRASIL, 1995). Esses contratos temporários não tinham amparo nem no Estatuto dos Servidores Municipais, nem na Consolidação das Leis de Trabalho (CLT). Foi promulgada uma lei específica para reger a situação dos novos profissionais, que procurava se apoiar na legislação federal para situações de emergência (DUQUE DE CAXIAS, 2005; BRASIL, 1993). A existência de dois regimes com diferentes remunerações, garantias e vantagens, além das insuficiências sobretudo do Regime Estatutário, são fatores que vieram a gerar insatisfação entre os profissionais e contribuíram para dificultar sua fixação na estratégia.

As condições de remuneração dos dois grupos permaneceram distintas; os estatutários recebiam as férias e o $13^{\circ}$ salário com base em seus salários originais, enquanto os contratados em caráter temporário recebiam ambas as remunerações sobre o valor total de seus vencimentos. Só houve concurso público para tentar regularizar a situação em 2008, sem muito sucesso até o momento em que este artigo foi escrito. 


\section{Perfil dos entrevistados}

A maioria dos 25 médicos entrevistados era composta de mulheres (76\%), com predomínio da faixa etária entre 31 a 40 anos (36\%) e estatutários (76\%). Com relação à trajetória no mercado de trabalho, o maior grupo (44\%) era constituído por médicos com mais de 20 anos de formados, sendo que $28 \%$ tinham entre 10 e 20 anos de formados e uma proporção equivalente com menos de dez anos de formação. Observou-se também que 36\% dos profissionais estava há mais de sete anos na estratégia. Uma elevada proporção dos entrevistados $(74 \%)$ acumulava a função de médico de família com outros vínculos de trabalho, fenômeno comum na classe médica.

Todos os entrevistados relataram ter formação especializada, com predomínio de pediatras (24\%), seguidos por outras especialidades como medicina do trabalho e endocrinologia (16\%), clínica médica e ginecologia/obstetrícia (12\%), gastroenterologia (8\%) e psiquiatria (4\%). A presença de médicos especialistas em medicina de família ainda era muito pequena (20\%) em relação às outras especialidades. $\mathrm{O}$ fato de não ser uma exigência para a atuação na estratégia, diferentemente de outras especialidades, contribuiu para o perfil encontrado atualmente(BRASIL, 2000). A criação de novos cursos de especialização específicos para Saúde da Família, pós-graduação, mestrado e/ou doutorado, e também a prova de titulação realizada pela Sociedade Brasileira de Medicina de Família e Comunidade, com apoio do Departamento de Atenção Básica, vêm contribuindo para a presença de alguns especialistas em MFC dentre os médicos atuantes no município, mas o quantitativo ainda é muito pequeno (SBMFC, 2006; 2007).

\section{Rotatividade}

Os dados da tabela 1, a seguir, mostram que apesar das constantes contrataçôes de médicos, o número de desligamentos foi aumentando no período analisado, passando de 26\% em 2005, para 54\% em 2008. Como se pode ver, $50 \%$ dos médicos são admitidos e outros 35\% são desligados em média a cada ano. No ano de 2008, com a realização do concurso público, que abriu 97 vagas para médicos de família, foram convocados em um primeiro momento 57 classificados, mas o número de desligamentos também aumentou, confirmando assim a alta rotatividade dos médicos da estratégia no município. 
Tabela 1. Situação das equipes com relação à permanência ou mudança dos profissionais de saúde (enfermeira e médico) nas equipes

\begin{tabular}{l|c|c}
\hline Situação da equipe & N & $\%$ \\
\hline Mesmos profissionais & 13 & 46,4 \\
\hline Mudaram os dois & 3 & 10,7 \\
\hline Mudou enfermeiro & 6 & 21,4 \\
\hline Mudou o medico & 6 & 21,4 \\
\hline Total & 28 & 100,0 \\
\hline
\end{tabular}

Fonte: Elaboração própria, a partir da análise dos dados coletados na pesquisa.

\section{As percepções dos médicos de família}

A análise das falas dos participantes do grupo focal mostrou grande insatisfação com o ambiente de trabalho. Os participantes se queixaram de que algumas equipes estavam instaladas em unidades básicas tradicionais de saúde e outras, em locais adaptados como Centros Integrados de Educação Pública (CIEPs), sedes de associações de moradores e sedes de igrejas. A análise revelou um forte sentimento de tristeza e angústia diante dos problemas físicos das unidades, especialmente com relação às condições sanitárias e a falta de conforto para trabalhar. Confirmando o resultado da análise dos questionários, $48 \%$ das respostas consideraram as estruturas pouco adequadas e $20 \%$ inadequadas.

Quanto aos serviços básicos das unidades, os participantes relataram que em diversas unidades não havia ligação adequada com serviços públicos básicos água, luz e telefonia - e ocorriam problemas constantes quanto ao abastecimento dos mesmos, o que prejudicava a realização das atividades. A ausência de telefones, ou computadores em rede, prejudicava a comunicação entre as equipes e a coordenação. Os profissionais reclamaram de ter de utilizar seus telefones celulares particulares para se comunicarem com a supervisão da estratégia e demais setores. Também ficou evidente a insatisfação quanto à ausência de equipamentos, materiais e insumos necessários à execução do trabalho.

A maioria dos profissionais considerava a carga de trabalho como pesada. Houve muita exaltação nas falas sobre a carga horária exigida dos médicos da estratégia em relação aos demais setores. Citaram o grande número de famílias a ser atendido como um fator de estresse no trabalho. Além da grande população 
atendida, em decorrência das normas da estratégia, os médicos se queixaram da carga de trabalho excedente resultante de demandas dos vereadores pelo atendimento de pessoas fora da população adscrita. A indicação política do pessoal administrativo das unidades, atuando como fiscais do horário e agenda dos médicos, foi outra queixa relatada por estes. A carga de trabalho excessiva, segundo os participantes, tinha a ver também com o grande número de atendimentos de demanda espontânea, que, segundo eles, ocorreria em função do desconhecimento da população do modelo de atenção da estratégia. Essa demanda interferiria negativamente na programação das atividades da equipe.

Houve muita controvérsia no grupo focal a respeito do modelo de trabalho na estratégia, baseado em equipe multiprofissional, em contraste com a opinião dos entrevistados $-44 \%$ dos médicos consideraram boa a relação com os membros da equipe e $40 \%$ como muito boa.

Foram marcantes nas falas dos profissionais os sentimentos de isolamento e solidão. $\mathrm{O}$ fato de muitas equipes trabalharem sozinhas dentro de uma unidade foi relatado como um elemento favorecedor desses sentimentos. Vários médicos relataram dificuldade para dialogar com colegas de profissão sobre casos clínicos, falta de espaço para trocar informações e experiências e até não haver momentos de confraternização entre colegas.

O tema que mais provocou indignação na fala dos profissionais foi a política municipal de recursos humanos. A remuneração praticada na estratégia foi considerada muito ruim e inadequada para uma carga horária de 40 horas semanais, em comparação a salários praticados em outros serviços de saúde e a inexistência de plano de cargos, carreira e salários adequados para o regime da estratégia. Outras questôes desta política, dos quais os médicos se queixaram, foram: a irregularidade das atividades de educação permanente e a dificuldade para dialogarem com os especialistas, quando têm dúvidas sobre casos clínicos mais complexos. Os participantes consideraram que os especialistas que deveriam dar suporte à Saúde da Família não entendem o modelo de atenção da estratégia e manifestariam desprezo ou descaso com os médicos de família, como se eles não tivessem seu valor.

Sobre as expectativas da carreira do médico de família, os participantes mostraram-se bastante pessimistas, colocando a situação encontrada atualmente no município desestimulante, sobretudo os baixos salários e a ausência de 
1302 plano de cargos e carreiras, que não dão perspectiva de futuro. Tais fatores os fazem pensar em desistir, segundo diversas falas. A falta de motivação, mágoas, decepções, tristeza, desilusão, insatisfação e falta de esperança foram percebidas claramente na fala dos profissionais durante a discussão.

Em síntese, a análise das falas revelou que os fatores que estimulam a continuidade do profissional na estratégia atualmente são o vínculo estabelecido com a comunidade, o reconhecimento, pelos usuários, do trabalho realizado e a identificação com a filosofia da estratégia. Alguns participantes justificaram sua continuidade no mesmo por estarem em fim de carreira, próximos da aposentadoria. Já entre os fatores que desestimulam a continuidade, podem-se destacar: ausência de plano de cargos, carreiras e salários; condiçôes físicas das unidades; carga elevada de trabalho; isolamento e dificuldade para obter suporte técnico de especialistas.

\section{Discussão}

A situação encontrada em Duque de Caxias tem clara relação com a fragilidade do quadro regulatório nacional, tanto para a formação de especialistas na área, quanto para o exercício da atividade, o que contrasta com a experiência de alguns países que priorizam a atenção primária nos seus sistemas públicos de saúde. A estratégia foi criada sem o apoio de um programa de residência médica. Só onze anos depois de seu funcionamento, o Ministério da Saúde passou a apoiar a formação especializada, que até hoje oferece um número reduzido de vagas, as quais nem sempre são totalmente preenchidas.

Até hoje, não há qualquer iniciativa governamental no sentido de planejar e regular o estoque de médicos e suas diferentes especialidades no Brasil, tal como ocorre em outros países. A frágil regulação deixa o controle da atividade médica nas mãos das sociedades especializadas, e contribui para estimular os profissionais a buscar no mercado as especialidades que permitem maiores ganhos financeiros e prestígio social. A medicina de família e comunidade não se encontra entre essas especialidades, o que a torna pouco atrativa. Pode-se dizer, neste sentido, que houve pouco investimento do Estado brasileiro tanto para a formação, quanto na regulação da atividade profissional, que constituem condições fundamentais para a sustentabilidade da estratégia, particularmente no que diz respeito à fixação dos profissionais médicos. Esta fragilidade dos instrumentos estatais de regulação 
da atividade é contraditória com a retórica oficial a respeito da importância da saúde da família, apresentada desde seu início como uma estratégia voltada para a reorientação do modelo assistencial do SUS e não apenas da atenção primária.

A pequena proporção de profissionais com formação na especialidade decorre da precariedade da política nacional para a formação da especialidade e da frágil regulação sobre as residências médicas em geral, cujo controle está principalmente com as sociedades médicas de especialistas (GIRARDI; CARVALHO, 2002). Há tanto uma pequena oferta de vagas para residentes em Saúde da Família, quanto pequena procura e incentivo para preenchimento das vagas existentes (CAMPOS, 2011; RIBEIRO, 2011). Como não há exigência de especialização para atuação na estratégia, nem diferenciação salarial entre os especialistas e não especialistas, a especialização fica em segundo plano para muitos profissionais. A situação brasileira é diferente da que prevalece em outros países, como se viu na introdução - na Espanha, por exemplo, há exigência de formação especializada para a atuação na área e possui amplo programa de residência médica para a mesma (ESPANHA, 2005).

Para enfrentar a carência de profissionais médicos, o Ministério da Saúde vem realizando encontros, debates e seminários para propor a fixação de profissionais na estratégia e em áreas remotas e de difícil acesso (BRASIL, 2011). Alguns já foram iniciados, para tentar resolver o problema por meio de uma série de portarias do ministério, como o cruzamento de dados no Cadastro Nacional de Estabelecimentos de Saúde, que impede que o profissional atue em diversos vínculos ao mesmo tempo e ultrapasse a jornada de trabalho permitida (BRASIL, 2011). Outra iniciativa, visando melhorar a adesão e inserção de profissionais médicos na estratégia, oferece abatimento de dívida na Estratégia de Financiamento Estudantil (FIES) ao graduado que atuar em áreas estipuladas pelo Ministério da Saúde (BRASIL, 2011). Recentemente, o ministério permitiu a flexibilização da carga horária para o médico na estratégia (BRASIL, 2011).

A partir de 2003, com o PROESF 1 e o Programa de Saúde Bucal, passou-se a estimular fortemente os municípios a expandirem o número de equipes, o que nem sempre foi feito com planejamento adequado, tal como ocorreu em Duque de Caxias. Grande parte dos problemas da estratégia no município se deve principalmente ao processo de implantação sem planejamento, que foi favorecido pelos estímulos federais para a expansão rápida do mesmo. Uma legislação 
1304 inadequada gerou dois regimes de contratação com direitos distintos e até conflitantes, além de contraditórios com a legislação federal e com o Estatuto dos Servidores Municipais. Foram criadas unidades sem condições físicas adequadas de funcionamento, a contratação de recursos humanos não obedeceu a critérios mínimos, desde a seleção até a adequação dos vínculos de trabalho. Tais aspectos estão relacionados aos interesses políticos que prevaleceram entre 2001 e 2008. A expansão rápida da estratégia entre 2005 e 2008, devido à agenda política local e favorecida pela política nacional - incentivos financeiros do PROESF, da Política Nacional de Atenção Básica e de Saúde Bucal - agravou o problema.

O problema encontrado no município também ocorreu em outros municípios, tendo inclusive motivado iniciativas do Governo Federal, visando melhorar as condições de trabalho e das unidades de saúde da família. Entrevista recente do diretor do Departamento de Atenção Básica (DAB) do Ministério da Saúde (SBMFC, 2011) menciona medidas visando corrigir as precárias condiçōes estruturais das UBS no país, explicitamente, o Programa de Requalificação das Unidades Básicas de Saúde, que visa dotar a atenção básica de unidades adequadas. Outras iniciativas do ministério neste sentido são o Plano Nacional de Implantação de Unidades Básicas de Saúde para Equipes de Saúde da Família (BRASIL, 2009), que destina recursos específicos para a construção de novas unidades, além de pesquisa em andamento sobre as condições de infraestrutura das UBS, que vem sendo realizada em todos os municípios (BRASIL, 2011).

As consequências da explosão do número de equipes de saúde da família nas condições mencionadas passaram a se manifestar no município de forma mais contundente a partir de 2009. Para tentar enfrentar os problemas gerados, houve necessidade de realização de novos concursos para tentar superar a rotatividade, construção de novas unidades e adequação de outras à legislação de vigilância sanitária. Como não houve mudança na legislação municipal de recursos humanos, vários dos problemas antigos relativos às condições de remuneração e direitos dos profissionais se agravaram. Desta forma, permanecem vínculos precários de trabalho, tal como em outros lugares do país (NESCOM, 2007; GIRARDI, 2010; JUNQUEIRA, 2010). A remuneração deficiente vem estimulando o acúmulo de vínculos de trabalho por parte dos médicos, que leva ao descumprimento da carga horária estipulada em prejuízo da qualidade do seu trabalho, problema que também ocorre em outros municípios do país (BRASIL, 2000; NEY, 2009). 
O presente estudo abordou os fatores que interferem na fixação do médico na estratégia, tendo por base pesquisa realizada no município de Duque de Caxias e relacionando-os com o débil quadro regulatório para a profissão médica, sobretudo para a especialidade de medicina de família e comunidade. A pesquisa mostrou que vários problemas enfrentados em Duque de Caxias e em outros municípios decorrem desta fragilidade regulatória.

A grande expansão da estratégia no município, a partir de 2005, não foi acompanhada nem de planejamento adequado, nem de disposições legais que facilitassem a gestão de seus recursos humanos. Como todos os profissionais envolvidos, o médico é o que tem maior poder de barganha, pelas alternativas que o mercado lhe oferece, e vem sendo difícil mantê-lo na estratégia, que apresenta rotatividade muito elevada no município.

A falta de preparação do município para a expansão da Estratégia Saúde da Família resultou em diversos problemas para a não fixação dos médicos, e o mais grave foi a não-estruturação da carreira de médico de família, que impediu uma perspectiva de futuro para os profissionais e os desestimulou a continuar na estratégia. Esse problema não ocorre apenas em Duque de Caxias, sendo uma realidade muito mais geral, como vários estudos mostram e vêm motivando iniciativas do Ministério da Saúde para tentar reduzir a precariedade dos vínculos de trabalho no SUS. Outros aspectos da política nacional para a estratégia foram associados à dificuldade para fixar os médicos, tais como a carga horária considerada elevada e o quantitativo excessivo de pacientes vinculados a uma equipe de saúde.

Problemas de infraestrutura também contribuem para a não-fixação dos profissionais, como condições inadequadas e até insalubres de unidades de saúde, falta de meios de comunicação e apoio logístico. A interferência política na gestão da estratégia e sobretudo das unidades foi outro problema sério citado na pesquisa e que seguramente não afeta apenas o município pesquisado, como outros estudos confirmam. A pesquisa revelou outros entraves à permanência do médico, relacionados com a gestão do sistema, dentre os quais a dificuldade de encaminhar pacientes para procedimentos de média e alta complexidade e a grande demanda espontânea de população não adscrita, decorrente da má gestão da rede de serviços. 
São poucos os fatores relatados que favorecem a permanência dos médicos. Todos eles estão relacionados a questões culturais e de valores pessoais, como a identificação com a filosofia da estratégia e o vínculo com as comunidades carentes. Alguns entrevistados mencionaram, inclusive, que se sentem culpados quando pensam em deixar o trabalho, porque deixariam as comunidades desassistidas. Embora alguns profissionais manifestem esse tipo de espírito missionário, não se pode contar com o mesmo para estruturar um programa nacional de grande importância, até porque ele não é tão comum.

Os resultados da pesquisa apontam claramente para a necessidade de estudos relativos às necessidades de regulação estatal sobre a formação e o exercício da profissão médica, em especial da saúde da família. Também é necessário que se conheçam mais as dificuldades para a definição de planos de carreira adequados no nível local e as possibilidades de implantação de normas federais mais rígidas, tanto para o controle da adoção de práticas inadequadas, quanto para o estímulo à regularização dos vínculos profissionais. ${ }^{1}$

\section{Agradecimentos}

À Organização Panamericana de Saúde (OPAS), pelo apoio através da bolsa de estudos para realização do Mestrado em Saúde da Família, que resultou neste artigo.

\section{Referências}

BARDIN, Laurence. Análise de Conteúdo. Lisboa: Edições 70, 2007. 223 p.

BLOOR, K.; MAYNARD, A. Planning human resources in health care: towards an economic approach, an international comparative review. Disponível em: <http://www.chsrf.ca/ PublicationsAndResources/ResearchReports/CommissionedResearch/03-03-01/a8ae7a7e79f3-47f5-9f65-92b9fe71b873.aspx>. Acesso em: 20 fev. 2011. 27 p.

BRASIL. Ministério da Saúde. Departamento de Atenção Básica. DAB lança questionário e convoca municípios a participar de pesquisa de avaliação (24/5/11). Disponível em: <http:// dab.saude.gov.br/noticia/noticia_ret_detalhe.php?cod=1275>. Acesso em: 09 ago. 2011.

BRASIL. Ministério da Saúde. Gabinete do Ministro. Portaria GM/MS n.o 1.444 de 28 de dezembro de 2000. Estabelece incentivo financeiro para a reorganização da atenção à saúde bucal prestada nos municípios por meio do Programa de Saúde da Família. Diário Oficial [da] República Federativa do Brasil, Brasília, DF, v. 0, n. 0, p. 85, col. 1, dez. 2000. Seção I. Portaria GM/MS no. 673, de 03 de junho de 2003. Atualiza e revê o incentivo financeiro às Ações de Saúde Bucal, no âmbito do Programa de Saúde da Família, parte 
integrante do Piso de Atenção Básica - PAB. Diário Oficial [da] República Federativa do Brasil, Brasília, DF, v. 0, n. 0, p. 44, col. 2, jun. 2003. Seção I.

. Portaria GM/MS No 2.226, de 18 de setembro de 2009. Institui, no âmbito da Política Nacional de Atenção Básica, o Plano Nacional de Implantação de Unidades Básicas de Saúde para Equipes de Saúde da Família. Diário Oficial [da] República Federativa do Brasil, Brasília, DF, v. 0, n. 0, p. 654, col. 1, set. 2009. Seção I.

Portaria GM/MS no. 1.377, de 13 de junho de 2011. Estabelece critérios para definição das áreas e regiōes prioritárias com carência e dificuldade de retenção de médico integrante de equipe de saúde da família oficialmente cadastrada e das especialidades médicas prioritárias de que tratam o inciso II e o $\$ 3^{\circ}$ do art. 6-B da Lei no 10.260 , de 12 de julho de 2001, no âmbito do Fundo de Financiamento ao Estudante do Ensino Superior (FIES) e dá outras providências. Diário Oficial [da] República Federativa do Brasil, Brasília, DF, v. 0, n. 0, p. 45, col. 2, jun. 2011. Seção I.

. Portaria GM/MS no 2.027, de 25 de agosto de 2011. Altera a Portaria no 648/GM/

MS, de 28 de março de 2006, na parte que dispóe sobre a carga horária dos profissionais médicos que compõem as Equipes de Saúde da Família (ESF) e na parte que dispõe sobre a suspensão do Piso de Atenção Básica (PAB Variável). Diário Oficial [da] República Federativa do Brasil, Brasília, DF, v. 0, n. 0, p. 90, col. 1, ago. 2011. Seção I.

BRASIL. Ministério da Saúde. Secretaria de Assistência à Saúde. Coordenação de Saúde da Comunidade. Saúde da Família: uma estratégia para a reorientação do modelo assistencial. Brasília: Ministério da Saúde, 1997. 36p.

BRASIL. Ministério da Saúde. Secretaria de Atenção à Saúde. Departamento de Atenção Básica. Política nacional de atenção básica. Brasília: Ministério da Saúde, 2006. 60 p.

Portaria SAS no. 134, de 4 de abril de 2011. Constitui responsabilidade dos gestores municipais, estaduais e do Distrito Federal/DF, bem como dos gerentes de todos os estabelecimentos de saúde na correta inserção, manutenção e atualização sistemática dos cadastros no SCNES dos profissionais de saúde em exercício nos seus respectivos serviços de saúde, públicos e privados. Diário Oficial [da] República Federativa do Brasil, Brasília, DF, v. 0, n. 0, p. 41, col. 2, abr. 2011. Seção I.

BRASIL. Ministério da Saúde. Secretaria de Gestão do Trabalho e Educação em Saúde. Seminário discute Provimento e Fixação de Profissionais em Áreas Remotas. Disponível em: <http:// portal.saude.gov.br/portal/saude/Gestor/visualizar_texto.cfm?idtxt=36830\&janela $=1>$. Acesso em: 21 out. 2011.

BRASIL. Ministério da Saúde. Secretaria de Políticas de Saúde, Departamento de Atenção Básica. Avaliação da implementação do Programa de Saúde da Família em dez grandes centros urbanos: síntese dos principais resultados. Brasília: Ministério da Saúde, 2005, 210 p.

Perfil dos médicos e enfermeiros do Programa Saúde da Família. V.1. Brasília: Ministério da Saúde, 2000. 146 p. 
BRASIL. Lei no ${ }^{\circ}$ 8.745, de 09 de dezembro de 1993. Dispõe sobre a contratação por tempo determinado para atender a necessidade temporária de excepcional interesse público, nos termos do inciso IX do art. 37 da Constituição Federal, e dá outras providências. Diário Oficial [da] República Federativa do Brasil, Brasília, DF, v. 0, n. 0, p. 18.937, dez. 1993. Seção I.

Lei Complementar no. 82, de 27 de março de 1995. Disciplina os limites das despesas com o funcionalismo público, na forma do art. 169 da Constituição Federal. Diário Oficial [da] República Federativa do Brasil, Brasília, DF, v. 0, n. 0, p. 4.277, mar. 1995. Seção I.

. Lei Complementar no. 96, de 31 de maio de 1999. Disciplina os limites das despesas com pessoal, na forma do art. 169 da Constituição. Diário Oficial [da] República Federativa do Brasil, Brasília, DF, v. 0, n. 0, p. 1, jun. 1999, Seção I.

. Lei Complementar no . 101, de 4 de maio de 2000 (Lei de Responsabilidade Fiscal).

Estabelece normas de finanças públicas voltadas para a responsabilidade na gestão fiscal e dá outras providências. Diário Oficial [da] República Federativa do Brasil, Brasília, DF, p. 1, mai. 2000, Seção I.

CAMPOS, C.; MALIK, A. Satisfação no trabalho e rotatividade dos médicos do Programa de Saúde da Família. Revista de Administração Pública. Rio de Janeiro, v. 42, n. 2, p. 347-68, mar-abr 2008.

CAMPOS, F.E.; HADDAD, A.E. et al. Programa de apoio à formação de médicos especialistas no Brasil (PRÓ-RESIDÊNCIA): indução para a formação de especialistas segundo necessidades do SUS. In: PIERANTONI, C.R.; DAL POZ, M.R.; FRANÇA, T. (Org.). O trabalho em saúde: abordagens quantitativas e qualitativas. Rio de Janeiro: Cepesc, 2011. p. 151-160.

CARLINI-COTRIM, B. Potencialidades da técnica qualitativa grupo focal em investigações sobre abuso de substâncias. Rev. Saúde Públ. São Paulo, v. 30, n. 3, p. 285-93, 1996.

DUQUE DE CAXIAS (Município). Lei no. 1.561, de 09 de fevereiro de 2001. Cria o regime Especial de Trabalho para atendimento ao Programa de Saúde da Família, e dá outras providências. Boletim Oficial [do] Município de Duque de Caxias, fev. 2001, n. 3112.

. Lei no 1.922 , de 08 de dezembro de 2005. Estabelece as necessidades de contratação temporárias de pessoal de excepcional interesse público e dá outras providências. Boletim Oficial [do] Município de Duque de Caxias, dez. 2005, n. 4990.

ESPANHA. Programa formativo de la especialidad de medicina familiar y comunitaria. Madri: Comisión Nacional de la Especialidad de Medicina Familiar y Comunitaria, 2005. 136 p. FORSTER, A.C. Estudo sobre a formação em Atenção Primária e Medicina de Família no Curso de Medicina da Universidad Autónoma de Madrid, Espanha, 1999/2000. Tese (Livredocência) - Ribeirão Preto: Universidade de São Paulo, 2004. 134 p. 
GÉRVAS, J. et al. Veinte años de reforma de la atención primaria en España, valoración para un aprendizaje por acierto / error. Madri: Red Española de Atención Primaria, 2005. 107 p. GIRARDI, S.N.; CARVALHO, C.L. Contratação e qualidade do emprego na Estratégia de Saúde da Família no Brasil. In: BARROS, A.F.R. (Org.). Observatório de recursos humanos no Brasil: estudos e análises. Rio de Janeiro: Fiocruz, 2003. p. 157-190.

GIRARDI, S.N.; CARVALHO, C.L. Mercado de trabalho e regulação das profissões de saúde. In: NEGRI, B.; FARIA, R.; VIANA, A.L.A. Recursos humanos em saúde, política, desenvolvimento e mercado de trabalho (Org.). São Paulo: Unicamp, 2002. p. 221-251.

GIRARDI, S.N. et al. O trabalho precário em saúde: tendências e perspectivas na Estratégia da Saúde da Família. Divulgação em Saúde para Debate. Rio de Janeiro, n. 45, p. 11-25, 2010. GIL, A.C. Métodos e técnicas de pesquisa social. São Paulo: Atlas, 1994. 207 p.

GOLDBERG, A. Innovaciones territoriales en el sistema de salud cubano, desde la revolucion hasta mediados de la decada de 1990. Scripta Nova, Revista Electrónica de Geografía y Ciencias Sociales. Barcelona, v. 41, n. 69, s/p, 2000. Disponível em: <http://www.ub.es/ geocrit/sn-69-41.htm>. Acesso em: 08 ago. 2010.

HASSEN, M.N.A. Grupos focais de intervenção no projeto sexualidade e reprodução. Brasil Horizontes Antropológicos. Porto Alegre, v. 8, n. 17, p. 159-177, 2002.

JUNQUEIRA, T.S. As relações laborais no âmbito da municipalização da gestão em saúde e os dilemas da relação expansão/precarização do trabalho no contexto do SUS. Cad. Saúde Pública. Rio de Janeiro, v. 26, n. 5, p. 918-928, 2010.

MACIEL FILHO, R.; PIERANTONI, C.R. O médico e o mercado de trabalho em saúde no Brasil: revendo conceitos e mudanças. In: BARROS, A.F.R. (Org.). Observatório de Recursos Humanos em Saúde no Brasil: estudos e análises. V.2. Rio de Janeiro: Fiocruz, 2004. p. 139-162. NÚCLEO DE EDUCAÇÃO EM SAÚDE COLETIVA. Universidade Federal de Minas Gerais. Precarização e qualidade do emprego na estratégia de saúde da família. Belo Horizonte: UFMG, 2007. 208 p.

NEY, M.S. Condições de fixação do médico na Estratégia Saúde da Família. 2009. 122 p. Dissertação (Mestrado em Saúde da Família) - Universidade Estácio de Sá, Rio de Janeiro, 2009.

ORGANISATION FOR ECONOMIC CO-OPERATION AND DEVELOPMENT. OECD Health Data 2011. Physicians by cathegory. Disponível em: <http://stats.oecd.org/ index.aspx?DataSetCode=HEALTH_STAT>. Acesso em: 05 set. 2011.

PISCO, L. Reform of Primary Health Care in Portugal (A journey through Family Medicine in Europe). Jornal Médico de Família. Lisboa, n. 151SE, p. 3, 2008.

RIBEIRO, M.A. Apontamentos sobre residência médica no Brasil. Brasília: Câmara dos Deputados (Consultoria Legislativa), 2011. 69 p. 
SEIXAS, P.H.; CORRÊA, A.N.; MORAES, J.C. Migramed - Migração Médica no Brasil: tendências e motivações. In: PIERANTONI, C.R.; DAL POZ, M.R.; FRANÇA, T. (Org.). O trabalho em saúde: abordagens quantitativas e qualitativas. Rio de Janeiro: Cepesc, 2011. p. 133-150.

SOCIEDADE BRASILEIRA DE MEDICINA DE FAMÍLIA E COMUNIDADE. $A$ especialização em medicina de família e comunidade e o desafio da qualificação médica para a Estratégia de Saúde da Família. SBMFC, nov. 2006. Disponível em: <www.sbmfc.org.br/ artigos>. Acesso em: 14 abr. 2008.

Desafios do ensino e da aprendizagem da atenção primária à saúde e da medicina de família e comunidade na graduação e pós-graduação em Medicina. Documento oficial da Sociedade Brasileira de Medicina de Família e Comunidade, 2007. Disponível em: <www. sbmfc.org.br>. Acesso: 13 abr. 2008.

Entrevista Hêider Aurélio Pinto em 21/06/11. Disponível em: <http://www.sbmfc. org.br/default.asp?site_Acao $=$ MostraPagina $\&$ PaginaId $=11 \&$ mNoti_Acao $=$ mostraNoticia \&noticiaId=374>. Acesso em: 09 ago. 2011.

VIANA, A.L.; DAL POZ, M. A reforma do Sistema de Saúde no Brasil e o Programa Saúde da Família Physis: Rev. saúde coletiva. Rio de Janeiro, v. 8, n. 2, p. 11-48, 1998.

\section{Nota}

${ }^{1}$ Os autores participaram igualmente de todos os processos de elaboração e construção do artigo. 
Critical factors for setting the physician in the Family Health Strategy

The paper analyzes the conditions that interfere with the setting of the physician in the Family Health Strategy. It is a qualitative research involving documentary analysis of human resources policies practiced, application and analysis of questionnaires and focus groups to identify the physicians' perception of their work in the strategy. There was a high turnover of doctors, determined by job dissatisfaction, poor working conditions and heavy workload. Among the characteristics of the human resources local politics stood out distortions in relation to remuneration, and problems with job plans, career and wages for family doctors, which limit and penalize the professional rise. The main reasons identified in the research that promote the continuity were identification with the strategy's philosophy, professional vocation and ability to serve the community. Reflections on human resources management in the strategy and SUS as a whole were presented, pointing out some of its main weaknesses, mostly including: deficiencies in vocational training policy for the specialty, insufficient regulation of the medical profession in Brazil by the State, and excessive political interference in the provision of public health services, particularly in this strategy.

> Key words: Family Health Strategy; family and community medicine; human resources in health; public health politics. 